\title{
Singularidades de Género nas Representações de Vinculação durante o Período Pré-Escolar
}

\author{
Singularities of Gender in the Representations of Attachment \\ during Pre-School Time
}

\author{
Joana Maia*, Manuela Veríssimo, Bruno Ferreira, Filipa Silva \& Marta Antunes \\ Instituto Superior de Psicologia Aplicada, Lisboa, Portugal
}

\begin{abstract}
Resumo
Visando identificar diferenças individuais no modo como as crianças encenam uma variedade de situações relacionadas com a vinculação, o Attachment Story Completion Task- ASCT (Bretherton. Ridgeway, \& Cassidy, 1990) tem sido utilizado em diferentes culturas, tanto com amostras clínicas como normativas, sendo uma das metodologias de narrativas mais utilizadas durante o período pré-escolar. Todavia, um ponto problemático da sua utilização prende-se com o facto de alguns estudos reportarem especificidades ao nível dos perfis narrativos evidenciados por meninos e meninas o que, do ponto de vista da Teoria da Vinculação, não seria de esperar. O ASCT foi aplicado a 252 crianças em idade pré-escolar $(M=62 ; D P=$ $15,1)$ tendo as narrativas sido analisadas através de uma escala contínua de segurança. Não foi encontrada nenhuma relação significativa entre a segurança das narrativas e o Q.I. verbal dos participantes. Nas respostas ao ASCT verificou-se uma diferença em função do género $[F(1,253)=11,8, p<0,01]$, com as histórias produzidas pelas raparigas, em média, a receberem pontuações mais elevadas na dimensão segurança. Várias hipóteses teóricas são exploradas para explicar os perfís encontrados e, partindo da ideia avançada por Oppenheim (1997) de que a aplicação deste tipo de metodologias representa uma situação indutora de ansiedade para a criança, é dado destaque à tese evolutiva proposta por Taylor et al. (2000) que afirma existir divergências ao nível das estratégias de regulação emocional tendencialmente adoptadas pelos dois sexos em situações de stress e de conforto interpessoal.

Palavras-chave: Representações de vinculação, diferenças de género, metodologias de narrativas.

Abstract

Attempting to identify individual differences in the way children tend to enact a variety of attachmentrelated scenarios, the Attachment Story Completion Task (Bretherton, Ridgeway, \& Cassidy, 1990) has been used in various cultures, both with normative and clinical samples, being considered a key narrative methodology in the field. Yet, a controversial question regarding its use is the fact that some studies unexpectedly report gender differences. Attachment Theory does not consider gender a relevant variable in the organization (quality) of attachment relationships. Gender is also not relevant when mental representations regarding such relationships are considered. Two hundred fifty two pre-schoolers $(M=62$; $D P=15.1)$ participated in this study. Children's narratives were coded according to a continuous security scale, and no correlation was found with Verbal I.Q. Gender differences were found $[F(1.253)=11.8, p<.01]$ with girls, on average, having higher scores than boys in all stories. Different reasons for children's play behavior were discussed, considering also that this kind of methodology can be a stressful situation to the child (Oppenheim, 1997). Special emphasis was given to Taylor et al. (2000) bio-evolutionist theory that explores the use of different strategies to cope with stress used by both genders.

Keywords: Attachment representations, gender differences, narratives.
\end{abstract}

\section{Comportamento de Vinculação e Género}

Em franco contraste com toda a atenção dada à qualidade dos cuidados prestados, às especificidades do

\footnotetext{
* Endereço para correspondência: Unidade de Investigação em Psicologia Cognitiva, do Desenvolvimento e da Educação, Instituto Superior de Psicologia Aplicada, Rua Jardim do Tabaco, 34, Lisboa, Portugal 1149-041. E-mail: jmaia@ispa.pt,mveriss@ispa.pt, raposoferreira@gmail.com e mantunes@ispa.pt
}

contexto sócio-familiar e às variáveis da própria criança, condicionantes que se pensa serem bases organizativas da maior ou menor segurança relacional estabelecida com os cuidadores, o género nunca foi considerado um factor especialmente saliente no contexto da Teoria da Vinculação. Guiando-se por uma lógica evolucionista, Bowlby $(1973,1980,1969 / 1982,1988)$ terá partido do pressuposto de que, face a situações de ameaça à sobrevivência, a existência de um objectivo comum de procura de segurança pela manutenção de proximidade ao cuidador 
deveria ter imposto às crias de ambos os sexos constrangimentos comportamentais semelhantes. Assim, com a ênfase a ser posta nos aspectos universais da teoria, ficou omissa, a possibilidade de poderem existir especificidades de género ao nível da segurança da vinculação e subsequente elaboração representativa.

Efectivamente, salvo raras excepções (e.g. Williams \& Blunk, 2003), a maioria dos estudos realizados durante a primeira infância e período pré-escolar sustentam a premissa da irrelevância do factor género afirmando, a este propósito, Bakermans-Kranenburg e van IJzendoorn (2009, p. 246) que "virtually no gender differences have been found in studies on infant attachment, which is a remarkable finding in itself." No entanto, é de notar que a maior parte dos estudos tende a recorrer a medidas de tipo comportamental (usualmente Situação Estranha (Ainsworth, Blehar, E. Waters, \& Wall, 1978) que incluem unicamente a mãe, verificando-se que, quando também o comportamento de vinculação ao pai é objecto de análise, o cenário tende a tornar-se mais complexo, com efeitos de interacção entre o género da criança e dos cuidadores a serem reportados (e.g. Schoppe-Sullivan et al., 2006). Alertando para a necessidade de mais estudos neste domínio, diferenças de género tanto ao nível da incidência das classificações de segurança e de insegurança (Barnett, Kidwell, \& Leung, 1998), como ao nível dos comportamentos discretos exibidos na Situação Estranha (David \& Lyons-Ruth, 2005) têm também sido registadas em estudos envolvendo populações de risco.

No que diz respeito à avaliação da qualidade das representações de vinculação na adolescência e idade adulta verificamos que não têm sido encontradas, pelo menos de forma sistemática, diferenças de género (c.f. Bakermans-Kranenburg \& van IJzendoorn, 2009) nos estudos envolvendo a Adult Attachment Interview (Hesse, 2008; Main \& Goldwyn, 1998). Também na maioria dos estudos realizados com as "Narrativas de Representação da Vinculação em Adultos" (H. S. Waters \& RodriguesDoolabh, 2004) não têm sido encontradas diferenças de género tanto durante a adolescência (e.g. Dykas, Woodhouse, Cassidy, \& H. S. Waters, 2006) como durante a idade adulta (e.g. Mikulincer, Shaver, Sapir-Lavid, \& Avihou-Kanza, 2009). No entanto, chamando a atenção para o facto de instrumentos semi-projectivos de tipo narrativo poderem ser mais susceptíveis para captar subtilezas de género, do que entrevistas de registo auto-biográfico excepções existem (e.g. Monteiro, Veríssimo, Vaughn, Santos, \& Bost, 2008) que alertam para a necessidade de que este aspecto seja melhor clarificado.

\section{Modelos Internos Dinâmicos - Uma Metáfora Conceptual}

Sabemos hoje que, ao longo do seu desenvolvimento, as crianças vão progressivamente internalizando os aspectos mais salientes das relações reais que estabelecem com os seus cuidadores, organizando representações mentais destas. Para explicar a associação entre o estabelecimento de ligações de proximidade emocional durante a infância, desenvolvimento e saúde mental, a Teoria da Vinculação (Bowlby, 1973, 1980, 1969/1982, 1988) apoia-se no conceito de Modelos Internos Dinâmicos (MID), uma "metáfora" conceptual utilizada para descrever componentes afectivos e cognitivos que formam representações mentais generalizadas e tendencialmente estáveis sobre o self, os outros e o mundo. Construídos activamente pela criança, no contexto de experiências interactivas que começam durante o primeiro ano de vida e que são repetidas praticamente de forma diária durante a infância, os MID operam primariamente a um nível sensório-motor emergindo precocemente sob a forma de expectativas rudimentares sobre a acessibilidade e responsividade das mesmas, variando na extensão em que são acessíveis à consciência.

A Teoria da Vinculação postula que o estabelecimento de uma relação de vinculação segura tem como correlato psicológico a organização de uma representação de vinculação segura, ou seja, de um modelo operacional de uma figura de vinculação específica que é perspectivada como estando disponível para a interacção, sendo capaz de proporcionar ajuda e conforto em caso de necessidade, mas que, simultaneamente, encoraja e permite os comportamentos de afastamento da criança que têm como objectivo a exploração do meio. Por sua vez, uma relação de vinculação insegura contribuirá para a emergência de um modelo operacional de uma figura de vinculação a quem se atribuem características tais como acessibilidade incerta, relutância em prestar ajuda e/ou reacções hostis prováveis.

Bowlby (1973) propõe que os MID são posteriormente actualizados pela integração de experiências relacionais relevantes, evoluindo para um conjunto organizado de crenças relativas a ser-se aceite, protegido, confortado e ajudado, quando necessário, por outros significativos (e vice-versa). É de realçar, contudo, a ênfase colocada na rejeição de qualquer determinismo linear, bem sinalizada pela adopção do termo dinâmicos que caracteriza os MID como estando abertos à revisão e mudança em virtude da experiência infirmatória de outras relações. No entanto, é também posta em realce a tendência para a sua continuidade esperando-se que, depois de consolidados, permaneçam relativamente estáveis ao longo da vida em virtude do forte impacto que têm para a própria percepção do real, guiando a experiência subjectiva. Neste sentido, os MID podem ser equiparados a filtros interpretativos, através dos quais os indivíduos absorvem e reconstroem novas experiências relacionais, de forma consistente com as experiências passadas, funcionando como modelo implícito para o estabelecimento de relações afectivas/intimas futuras (ver Bretherton \& Munholland, 2008). 
Maia, J., Verissimo, M., Ferreira, B., Silva, F. \& Antunes, M. (2012). Singularidades de Género nas Representações de Vinculação durante o Período Pré-Escolar.

\section{Attachment Story Completation Task}

Nos últimos vinte anos um dos esforços dos estudiosos da vinculação tem sido o de validar instrumentos capazes de captar os distintos níveis de análise em que este constructo pode ser estudado adequados a diferentes momentos desenvolvimentais. Mais recentemente, a atenção dos investigadores começou a deslocar-se do nível comportamental para o nível representacional vindo a verificar-se um aumento significativo na utilização de metodologias que elicitam narrativas (e.g. Attachment Doll-Play Interview; Oppenheim, 1997); The Manchester Attachment Story Task (Green, Stanley, Smith, \& Goldwyn, 2000) como uma forma legítima de aceder ao "mundo interno representacional" de crianças em idade pré-escolar e escolar (ver Emde, 2003).

Combinando a play therapy de Donald Winnicott (1971) e a tradição dos métodos projectivos com uma abordagem psicométrica, o ASCT apresenta boas taxas de concordância com medidas que avaliam o comportamento de vinculação da criança à mãe durante a primeira infância (e.g. Bretherton, Ridgeway, \& Cassidy, 1990; GlogerTippelt, Gomille, Köenig, \& Vetter, 2002; Silva et al., 2008) assim como com a qualidade das representações de vinculação maternas (e.g., Gloger-Tippelt et al., 2002; Miljkovitch, Pierrehumbert, Bretherton, \& Halfon, 2004). Contudo, mais estudos são necessários para legitimar a sua validade convergente e discriminante, aspecto que é dificultado pelo facto de o instrumento não obrigar à utilização de um sistema de classificação único, existindo a possibilidade de ajustar a escolha das histórias e o processo de codificação aos objectivos específicos da investigação em curso.

Neste contexto, um aspecto polémico prende-se com o facto de alguns estudos terem reportado diferenças nos perfis narrativos tipicamente adoptados por meninas e meninos o que levanta questões importantes sobre a validade deste tipo de metodologias uma vez que, do ponto de vista da Teoria da Vinculação, estas diferenças não seriam de esperar. Numa amostra normativa de pré-escolares Verschueren, Marcoen e Schoefs (1996) registaram que, embora o género não estivesse associado de forma significativa com a classificação de vinculação atribuída, quando era feita uma avaliação quantitativa das histórias as meninas pontuavam significativamente mais alto na escala de segurança. Comparando uma amostra de crianças portuguesas com uma amostra de crianças norte-americanas, Wong et al. (2011) verificaram que nas duas amostras estas tendiam a construir histórias mais elaboradas e que apresentavam melhor organização causal e temporal.

Estudando uma amostra de filhos de mães com historial, crónico ou transitório, de depressão Trapolini, Ungerer e McMahon (2007) reportaram que as raparigas tendiam a incluir nas suas histórias mais temas de educação autoritária (e.g. castigo, disciplina severa), enquanto os rapa- zes tendiam a incluir mais eventos bizarros que aparecem de forma desconexa ao longo das histórias.

Também estudos com famílias mono parentais (GlogerTippelt \& Konig, 2007) e em situação de pós-divórcio (Page \& Bretherton, 2001, 2003a, 2003b) têm identificado maiores índices de insegurança e de desorganização nas narrativas dos rapazes, tendendo estes a incluir significativamente menos comportamentos pró-sociais e a apresentar uma maior preponderância de conteúdos agressivos e violentos. Por sua vez, as raparigas encenam um maior número de interacções sociais positivas, nomeadamente, manifestações de afecto e de reparação após conflito. Finalmente, no maior estudo que até à data se focou especificamente na questão do género comparando narrativas ASCT produzidas em 5 diferentes países, cotadas através de um procedimento de Q-Sort (Pierrehumbert et al., 2009), verificaram que as raparigas expressavam um maior número de representações de vinculação seguras no contexto da interacção pais-criança encenada, acedendo também mais facilmente a conteúdos de ordem emocional e sendo mais coerentes na sua articulação. É de notar que o mesmo padrão de resultados tem sido encontrado com tarefas de completamento de histórias de âmbito mais geral que envolvem histórias evocativas de conteúdos relativos à dinâmica familiar e ao funcionamento interpessoal da criança (e.g., Von Klitzing, Kelsay, Emde, Robinson, \& Schmitz, 2000).

Dando continuidade à investigação, até ao momento, realizada com o ASCT na população portuguesa, (e.g. Benavente, Justo, \& Veríssimo, 2009; Carvalho et al., 2010; Custódio \& Cruz, 2008; Maia, Ferreira, Veríssimo, Santos, \& Shin, 2008; Silva et al., 2008; Torres, Maia, Veríssimo, Fernandes, \& Silva, 2011), o presente estudo teve como principal objectivo averiguar a existência de diferenças significativas de género na performance de uma amostra normativa de crianças em idade pré-escolar.

\section{Método}

\section{Participantes}

Participaram neste estudo 230 crianças (113 meninos e 117 meninas), oriundas de instituições de ensino pré-escolar do Distrito de Lisboa. No momento da avaliação tinham idades compreendidas entre os 36 e os 71 meses $(M=55,42, D P=12,76)$, a idade das mães variava entre os 22 e os 47 anos $(M=34,71 ; D P=5,03)$ e a dos pais entre os 23 e os $55(M=37,35 ; D P=5,71)$. O nível de educação materno variou entre os 4 e os 23 anos de escolaridade $(M=11,70 ; D P=4,43)$, o paterno entre os 4 e os 19 $(M=11,09 ; D P=5,57)$, pertencendo as famílias a um nível socioeconómico médio e médio-alto.

\section{Instrumentos}

Representações de Vinculação. O ASCT consiste numa entrevista de cerca de 30 minutos, aplicável entre os $3 \mathrm{e}$ os 6 anos, durante a qual, com a ajuda de uma família de 
bonecos, são apresentados 5 inícios de histórias ligadas a cenários relevantes no contexto das experiências de vinculação pais-filhos. É então pedido à criança que lhes dê continuidade, ilustrando as interacções entre as personagens. Cada instrução foi construída de modo a evocar uma problemática distinta: figura de vinculação em situação de autoridade face a um percalço acidental da criança; activação do sistema de vinculação e resposta parental à dor e ao medo da criança; ansiedade de separação e capacidade de coping com um cuidador substituto e tonalidade afectiva da reunião familiar. Uma instrução adicional neutra é administrada inicialmente, para assegurar que a criança compreende o procedimento, mas não é cotada.

Competência Linguística. De forma a controlar potenciais efeitos ao nível das narrativas produzidas por rapazes e raparigas decorrentes de diferenças ao nível da capacidade lexical e da compreensão verbal foram aplicados os sub-testes verbais da versão revista da Wechsler Preschool and Primary Scale of Intelligence WPPSI-R - de Wechsler (1989, aferida para a população portuguesa por Seabra-Santos et al., 2003).

\section{Procedimento}

Ambos os instrumentos foram aplicados de forma individual, em ocasiões distintas, por membros independentes da equipa de investigação. As entrevistas do ASCT foram gravadas em vídeo e posteriormente analisadas tendo como referencial uma escala contínua de segurança (Maia, Ferreira, Silva, Fernandes, \& Veríssimo, 2009) que sintetiza procedimentos de codificação provenientes de diferentes abordagens. Para cada narrativa, ao longo de uma escala de 8 pontos, é avaliada a extensão em que o sujeito resolve coerentemente o problema apresentado, a positividade da representação parental e o conhecimento emocional que revela. São também tidos em conta parâmetros associados ao processo narrativo, nomeadamente, emoção geral expressa, comportamento não verbal, fluência do discurso, grau de investimento na tarefa e qualidade da interacção com o entrevistador. Todas as narrativas foram cotadas por dois investigadores $(50 \%$ das narrativas cotadas por investigadores do sexo feminino, $50 \%$ cotadas por um investigador do sexo masculino e por outro do sexo feminino) previamente treinados, estranhos à situação de recolha dos dados, bem como a qualquer outra informação sobre as crianças. Os valores de correlação entre os dois investigadores (calculado como correlações intra classes) variaram entre 0,73 e 0,98 , com $91 \%$ dos valores a situarem-se dentro de 1 ponto na escala de $1-8$. Quando considerado em termos exactos de acerto/não acerto, o acordo entre os investigadores oscilou entre 0,56 e 0,74 (Kappa). Para cada história, o valor final foi obtido através da média das pontuações dadas pelos dois investigadores.

\section{Resultados}

A caracterização descritiva das pontuações de segurança encontradas para cada história é dada na Tabela 1.

Tabela 1

Valores Mínimos, Máximo, Médio e Desvio-Padrão (DP), Assimetria (Sk) e Achatamento(Ku) da Segurança em Cada História ( $n=252)$

\begin{tabular}{lrrrrrr}
\hline Histórias ASCT & Min & Max & $M$ & $D P$ & Sk & $\mathrm{Ku}$ \\
\hline Sumo entornado & 1 & 8 & 5,20 & 1,15 & $-0,63$ & 0,69 \\
Joelho magoado & 1 & 8 & 5,22 & 1,39 & $-0,49$ & $-0,33$ \\
Monstro no quarto & 1 & 8 & 5,51 & 1,25 & $-0,90$ & 0,82 \\
Partida & 1 & 7,67 & 5,08 & 1,26 & $-0,53$ & 0,21 \\
Reencontro & 1 & 8 & 5,19 & 1,50 & $-0,82$ & 0,13 \\
\hline
\end{tabular}

Correlações de Pearson entre as pontuações obtidas por cada sujeito nas diferentes histórias mostraram que estas se encontram significativamente relacionadas entre si ( $r$ entre 0,59 e 0,$74 ; p<0,001)$. O valor do alfa de Cronbach's para o conjunto das cinco histórias foi de 0,87 . Estes resultados permitiram combinar os valores das 5 histórias num valor único de Segurança $(M=5,24$; $D P=1,14$ )

As correlações entre cada uma das histórias, o Q.I. verbal e a idade da criança não apresentaram correlações significativas. A Segurança Total não evidenciou associação significativa com o Q.I. Verbal $(r=0,14 ; p=0,16)$ nem com a idade $(r=0,12 ; p=0,17)$.

Finalmente a idade da mãe, a idade do pai assim como as respectivas habilitações literárias não apresentaram correlações significativas com o valor de segurança total da criança.

\section{Diferenças em Função do Género}

Não foi registada nenhuma diferença significativa entre os dois géneros em termos de idade, $F(1,229)=1,38$, $p=0,24$, ou de QI verbal, $F(1,250)=1,38, p=0,24$.

Depois de validados os pressupostos da homocedasticidade com o teste de Levene $F(1,229)=1,38, p=0,24$ e da normalidade nas pontuações médias obtidas pelas raparigas e pelos rapazes [respectivamente, $\mathrm{KS}(115)$ $=0,07, p=0,2$ e $\operatorname{KS}(115)=0,06, p=0,2$, foram analisados efeitos de género através de testes $t$-student. A Figura 1 ilustra a caracterização gráfica das pontuações totais de segurança obtidas pelas raparigas $(M=5,48 ; D P=1,04) \mathrm{e}$ 
Maia, J., Verissimo, M., Ferreira, B., Silva, F. \& Antunes, M. (2012). Singularidades de Género nas Representações de Vinculação durante o Período Pré-Escolar.

pelos rapazes $(M=5,00 ; D P=1,18)$ assim como os valores obtidos em cada uma das histórias As meninas pontuaram significativamente mais alto que os meninos no valor global de segurança $t(229)=3,44, p<0,01$. A análise de cada uma das histórias confirmou estes resultados, o género feminino apresenta nas cotações da história sumo entornado $t(229)=2,07, p<0,05$, joelho magoado $t(229)=2,19, p<0,05$, monstro no quarto $t(229)=1,92$, $p<0,05$, partida $t(229)=2,43, p<0,05$ e reencontro $t(229)=3,43, p<0,01$, sempre valores mais elevados.

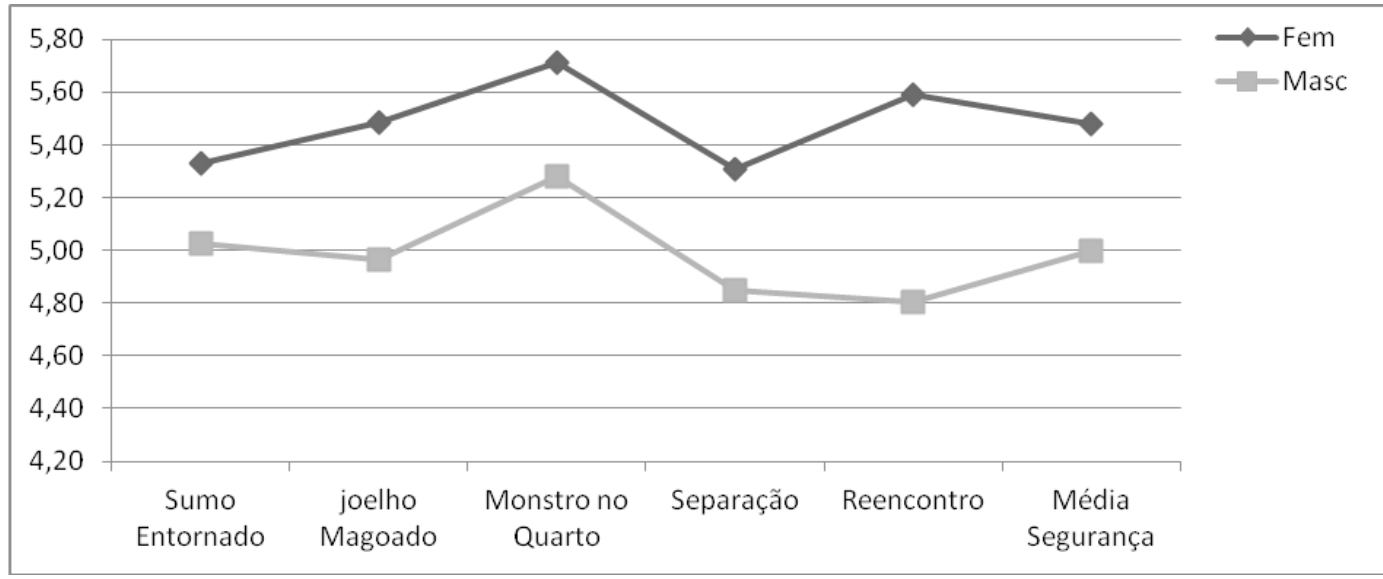

Figura 1. Caracterização gráfica das pontuações obtidas por meninos e meninas na média da segurança e nas diferentes histórias do ASCT.

\section{Discussão}

O facto de o ASCT carecer de um sistema de cotação único tem impossibilitado a criação de valores normativos de referência que possam auxiliar na interpretação das pontuações médias de segurança encontradas no nosso estudo, mediante comparação com outras populações previamente estudadas. No entanto, é de realçar que tanto as pontuações médias de meninos e de meninas se inscrevem no domínio da segurança, situando-se entre os pontos da escala que descreve representações pouco seguras (valor 5) e algo seguras (valor 6), como seria de esperar sendo esta uma população normativa. Os nossos resultados foram ao encontro dos dados usualmente reportados na literatura relativos à melhor performance narrativa evidenciada pelas raparigas em tarefas de completamento de histórias evocativas de conteúdos associados à vinculação. Neste estudo, em todas as histórias as raparigas, em média, pontuaram significativamente mais alto na escala de segurança. Tal significativa que os valores médios das raparigas, por comparação com os dos rapazes, se caracteriza pela narração de histórias relevantes do ponto de vista da vinculação mais elaboradas e coerentes, sendo feito um maior investimento na tarefa, pautado por maior facilidade e espontaneidade discursiva. Possivelmente, poderá também ter contribuído para estes valores a apresentação de um leque mais variado de afectos apropriados à situação narrada, a par com uma maior evidência de conhecimento emocional.

Até ao momento, várias hipóteses explicativas, com diferentes implicações práticas e teóricas, têm sido avançadas procurando explicar de que modo características associadas ao género poderão contribuir para algumas especificidades apresentadas nas narrativas de vinculação. Uma das mais difundidas prende-se com a possibilidade de existirem discrepâncias de género ao nível da competência verbal, variável que nem sempre é aferida. Mais difícil de controlar é a competência narrativa, ou seja, a capacidade de criar um enredo e de contar uma história. Evidenciando as raparigas, precocemente, um acesso mais espontâneo a conteúdos de ordem emocional e relacional (Butler \& Shalit-Naggar, 2008), tal poderá justificar que construam histórias mais coerentes e ricas em detalhes intra e inter-subjectivos.

Algumas evidências existem de que aspectos culturais associados à socialização podem também desempenhar um papel importante nesse sentido. No estudo de Pierrehumbert et al. (2009) foram comparados resultados de 5 países diferentes e em todos as raparigas foram identificadas como produzindo melhores narrativas. No entanto, a magnitude das diferenças foi agravada nos dois países hispânicos (Espanha e Chile), cuja micro-cultura familiar apoia ainda hoje, tal como em Portugal, a diferenciação dos papéis de género. Contudo, se a tendência das raparigas para apresentarem uma melhor performance resultasse exclusivamente de aspectos associados à capacidade verbal ou à competência narrativa, seria de esperar, por exemplo, que quando o primeiro factor é controlado, como é o caso da presente investigação, ou quando são utilizados sistemas de cotação que não englobam a coerência e a elaboração discursiva, como é o caso dos sistemas de análise de temas, ou baseados em critérios, estas tendência se esbatesse, o que nem sempre é o caso (e.g. Gloger-Tippelt \& König, 2007; Page \& Bretherton, 2001, 2003a, 2003b). No entanto, outro cenário possível é que os resultados encontrados reflictam reais diferen- 
ças ao nível da segurança das representações, o que, a ser confirmado, suscitará sem dúvida reflexões teóricas de maior vulto. Seguramente, uma delas terá de envolver a ponte, recentemente criada, (e.g., Bost et al., 2006) entre os teóricos da vinculação e aqueles que se debruçam sobre as ligações entre memória autobiográfica e linguagem, confirmando um dos pressupostos base da teoria relativo ao facto de, para além da primeira infância, a existência de uma comunicação criança-cuidador caracterizada pela abertura emocional constituir um dos pontos-chave para a estruturação e manutenção de modelos de vinculação seguros (Bowlby, 1973, 1980). Neste sentido, têm de ser analisados com atenção resultados (e.g. Fivush, Reese, \& Haden, 2006) que mostram que tanto os pais como as mães, quando solicitados a iniciar diálogos sobre eventos passados, carregados emocionalmente, são significativamente mais elaborativos e coerentes, integrando mais emoções e falando sobre a causualidade das mesmas, com as filhas, o que poderá justificar que estas construam representações de vinculação mais seguras.

Parece-nos igualmente que uma perspectiva merecedora de atenção diz respeito à hipótese de as referidas diferenças poderem reflectir, essencialmente, divergências ao nível das estratégias de regulação emocional tendencialmente adoptadas pelos dois sexos. Tem vindo a ser sugerido que a aplicação do ASCT pode ser perspectivada como uma situação indutora de ansiedade (ver Oppenheim, 1997), na medida em que cada instrução confronta a criança com um tema relacional complexo, conflituoso e emocionalmente carregado, com a aç̧ão a ser deixada no seu clímax. É-lhe então solicitado que, regulando a tensão emocional suscitada pela instrução, resolva de forma coerente o problema apresentado, ao mesmo tempo que se mantém em interacção com um adulto desconhecido. Na continuidade deste pensamento, fará sentido explorar a tese proposta por Taylor e colaboradores (c.f. Taylor et al., 2000; Taylor et al., 2002) que sugere uma predominância de estratégias comportamentais de tipo afiliativo no sexo feminino, caracterizadas pela procura e/ou prestação de cuidados e de protecção ("tend-or-befriend" no original), por oposição à predominância de estratégias comportamentais de luta ou de fuga ("fight-or-flight") no género masculino face a situações de stress e de confronto interpessoal.

Numa óptica evolucionista, a adopção de tais estratégias teria na sua base distintas vantagens adaptativas. Em situações adversas, as respostas de tipo afiliativo, ao optimizarem a criação e a manutenção de redes sociais, teriam sido mais adequadas para as fêmeas no mundo natural, favorecendo não só a promoção da segurança pessoal mas, também, assegurando a protecção das crias. Ao mesmo tempo, a consequente redução do stress permitida por este tipo de estratégias potenciaria a segregação de níveis mais elevados de oxitocina, hormona que desempenha um papel central no sistema de prestação de cuidados. Por sua vez, nas mesmas condições de stress, dotados de condições físicas de força e agilidade tendencialmente superiores às das fêmeas, os machos poderiam ter beneficiado de estratégias reactivas, de luta e de confronto, ou de reacções de fuga.

Um dos estudos no campo da vinculação que, até à data, mais parece estar em consonância com esta hipótese foi realizado por David e Lyons-Ruth (2005). Na análise de Situações Estranhas de um grupo de crianças em situação de negligência e/ou maus-tratos maternos estes investigadores identificaram um maior número de rapazes com uma vinculação desorganizada - insegura de tipo controlador, classificação apoiada pela combinação contraditória, aquando da reunião, de comportamentos pronunciados de evitamento - fuga (esconder debaixo de cadeira, correr para longe da mãe, imobilização motora, encolherem-se no chão) e de zanga - resistência dirigidos à mãe. Por seu turno, mesmo quando a conduta materna se tornava claramente inadequada ou ameaçadora, as raparigas procuraram em maior escala a proximidade física das mães embora, ao fazê-lo, apresentassem simultaneamente comportamentos indiciadores de medo (movimentos prudentes e hesitantes, imobilização motora), o que contribuiu para um maior número de classificações de vinculação desorganizada - segura.

Uma questão fundamental será, então, apurar até que ponto a partir de tais pontuações pode efectivamente, ser inferida maior segurança representacional, ou se estas podem ter carácter ilusório mascarando aspectos de insegurança relacional. Tem sido evidenciado que nas narrativas, especialmente no caso das raparigas, a presença per se de conteúdos positivos, de natureza pró-social ou respostas empáticas dirigidas aos pais pode não reflectir necessariamente uma representação de vinculação segura, podendo esta inclusão representar antes uma tentativa para excluir defensivamente conteúdos negativos, de forma a melhor lidar com a ansiedade suscitada pela tarefa. Alargando esta ideia, um dos poucos estudos exploratórios que procurou relacionar a coerência e a qualidade emocional das narrativas produzidas com aspectos do próprio processo narrativo mostrou que nem a presença de emoções positivas na trama da história, nem a presença de afectos positivos na expressão facial da criança se correlacionam linearmente com a coerência narrativa e com a qualidade de resolução do problema da história, com esta ausência de correspondência a parecer agravar-se no caso das raparigas (Ferreira, Maia, Pinto, Santos, \& Fernandes, 2010).

Pensamos que, como sugerido por Zanh-Waxler, Shirtcliff e Marceau (2008), uma preocupação da investigação futura, deverá ser clarificar a efectiva importância das diferenças encontradas para o ajustamento social e emocional dos sujeitos. Recorrendo a uma forma revista do ASCT, adaptada para situações de divórcio, este trabalho tem sido iniciado por Page e Bretherton (2001, 2003a, 2003b), com resultados preliminares surpreendentes. Até ao momento, a conclusão mais importante a retirar é que a presença de iguais conteúdos 
Maia, J., Verissimo, M., Ferreira, B., Silva, F. \& Antunes, M. (2012). Singularidades de Género nas Representações de Vinculação durante o Período Pré-Escolar.

nas narrativas de rapazes e de raparigas pode ter correlatos externos distintos e mesmo contraditórios, em termos de competência social, quando esta é avaliada pelas educadoras.

Afigura-se, deste modo incompleta a abordagem tradicional que espera que conteúdos representacionais positivos ou negativos predigam, linearmente e por si só, a adopção de comportamentos, respectivamente, pró e anti sociais por parte da criança. Parece ser necessário, sim, que o diagnóstico de positividade ou negatividade dado a estes conteúdos tenha em consideração o facto de os mesmos poderem ser influenciados pela socialização, nomeadamente por aquilo que, em cada idade (ver Granot \& Mayseless, 2001) e em cada cultura, corresponde ou se distancia do estereótipo de género. Do exposto, pensamos ficar clara a necessidade de serem realizados mais estudos que, controlando aspectos linguísticos e avaliando, de forma simultânea, especificidades de conteúdo, coerência narrativa e aspectos do processo narrativo, se foquem especificamente nesta temática. Só assim se poderão encontrar reunidas as condições para uma utilização responsável e fecunda deste tipo de metodologia.

\section{Referências}

Ainsworth, M., Blehar, M., Waters, E., \& Wall, S. (1978). Patterns of attachment: A psychological study of the strange situation. Oxford, UK: Lawrence Erlbaum.

Bakermans-Kranenburg, M., \& van IJzendoorn, M. (2009). The first 10,000 adult attachment interviews: Distributions of adult attachment representations in clinical and non-clinical groups. Attachment \& Human Development, 11(3), 223-263.

Barnett, D., Kidwell, S., \& Leung, K. (1998). Parenting and preschooler attachment among low-income urban African American families. Child Development, 69(6), 1657-1671.

Benavente, R., Justo, J., \& Veríssimo, M. (2009). Os efeitos dos maus-tratos e da negligência sobre as representações da vinculação em crianças de idade pré-escolar. Análise Psicológica, 27(1), 21-31.

Bost, K., Shin, N., McBride, B., Brown, G., Vaughn, B., Coppola, G., et al. (2006). Maternal secure base scripts, children's attachment security, and mother-child narrative styles. Attachment \& Human Development, 8(3), 241-260.

Bowlby, J. (1973). Attachment and loss: Vol. 2. Separation, anxiety, and anger. New York: Basic Books.

Bowlby, J. (1980). Attachment and loss: Vol. 3. Loss. New York: Basic Books.

Bowlby, J. (1982). Attachment and loss: Vol.1. Attachment $\left(2^{\text {nd }}\right.$ Rev. Ed.) New York: Basic Books. (Original work published 1969).

Bowlby, J. (1988). A secure base: Parent-child attachment and healthy human development. New York: Basic Books.

Bretherton, I., \& Munholland, K. (2008). Internal working models in attachment relationships: Elaborating a central construct in attachment theory. In J. Cassidy \& P. R. Shaver (Eds.), Handbook of attachment: Theory, research, and clinical applications ( $2^{\text {nd }}$ ed., pp. 102-127). New York: Guilford Press.
Bretherton, I., Ridgeway, D., \& Cassidy, J. (1990). Assessing internal working models of the attachment relationship: An attachment story completion task for 3-year-olds. In M. Greenberg, D. Cicchetti, \& E. M. Cummings (Eds.), Attachment during the preschool years: Theory, research, and intervention (pp. 273-308). Chicago, IL: University of Chicago Press.

Butler, R., \& Shalit-Naggar, R. (2008). Gender and patterns of concerned responsiveness in representations of the motherdaughter and mother-son relationship. Child Development, 79(4), 836-851.

Carvalho, M., Martins, C., Martins, E., Osório, A., Tereno, S., \& Soares, I. (2010, fev.). (Des) Continuidade entre a qualidade da vinculação na $1^{a}$ infância e representação da vinculação aos 3 anos. Trabalho apresentado no VII Simpósio Nacional de Investigação em Psicologia, Braga, Portugal.

Custódio, S., \& Cruz, O. (2008). As representações mentais das crianças acerca das figuras parentais. Psicologia: Teoria e Pesquisa, 24(4), 393-405.

David, D., \& Lyons-Ruth, K. (2005). Differential attachment responses of male and female infants to frightening maternal behaviour: Tend or befriend versus fight or flight? Infant Mental Health Journal, 21(1), 1-18.

Dykas, M. J., Woodhouse, S. S., Cassidy, J., \& Waters, H. S. (2006). Narrative assessment of attachment representations: Links between secure base scripts and adolescent attachment. Attachment \& Human Development, 8, 221-240.

Emde, R. (2003). Early narratives: A window to the child's inner world. In R. Emde, D. Wolf, \& D. Oppenheim (Eds.), Revealing the inner worlds of young children: The MacArthur Story Stem Battery and parent-child narratives (pp. 3-26) New York: Oxford University Press.

Ferreira, B., Maia, J., Pinto, A., Santos, A. J., \& Fernandes, C. (2010). Qualidade dos modelos internos de vinculação e a expressividade emocional em crianças de idade pré-escolar. Trabalho apresentado no VII Simpósio Nacional de Investigação em Psicologia, Braga, Portugal.

Fivush, R., Reese, E., \& Haden, C. (2006). Elaborating on elaborations: Role of maternal reminiscing style in cognitive and socioemotional development. Child Development, 77(6), 1568-1588. doi: 10.1111/j.1467-8624.2006.00960.x

Gloger-Tippelt, G., \& König, L. (2007). Attachment representations in 6-year-old children from one and two parent families in Germany. School Psychology International, 28(3), 313-330. doi: 10.1177/0143034307078540

Gloger-Tippelt, G., Gomille, B., König, L., \& Vetter, J. (2002). Attachment representations in 6-year-olds: Related longitudinally to the quality of attachment in infancy and mothers' attachment representations. Attachment \& Human Development, 4(3), 318-339.

Granot, D., \& Mayseless, O. (2001). Attachment security and adjustment to school in middle childhood. International Journal of Behavioral Development, 25(6), 530-541.

Green, J., Stanley, C., Smith, V., \& Goldwyn, R. (2000). A new method of evaluating attachment representations in young school-age children: The Manchester Child Attachment Story Task. Attachment \& Human Development, 2(1), 48-70. doi: $10.1080 / 146167300361318$

Hesse, E. (2008). The Adult Attachment Interview: Protocol, method of analysis, and empirical studies. In J. Cassidy \& P. R. Shaver (Eds.), Handbook of attachment: Theory, research, and clinical applications ( $2^{\text {nd }}$ ed., pp. 552-598). New York: Guilford Press. 
Maia, J., Ferreira, B., Silva, F., Fernandes, M., \& Veríssimo, M. (2009). Adaptação portuguesa do Attachment Story Completion Task - Manual de aplicação e cotação: Dimensão Contínua de Segurança. Manuscrito não-publicado, Instituto Superior de Psicologia Aplicada, Lisboa, Portugal.

Maia, J., Ferreira, B., Veríssimo, M., Santos, A. J., \& Shin, N. (2008). Auto-conceito e representações da vinculação no período pré-escolar. Análise Psicológica, 3(26), 423-433.

Main, M., \& Goldwyn, R. (1998). Adult attachment scoring and classification system. Unpublished manuscript, University of California at Berkeley, CA.

Mikulincer, M., Shaver, P., Sapir-Lavid, Y., \& Avihou-Kanza, N. (2009). What's inside the minds of securely and insecurely attached people? The secure-base script and its associations with attachment-style dimensions. Journal of Personality and Social Psychology, 97(4), 615-633.

Miljkovitch, R., Pierrehumbert, B., Bretherton, I., \& Halfon, O. (2004). Associations between parental and child attachment representations. Attachment \& Human Development, 6(3), 305-325.

Monteiro, L., Veríssimo, M., Vaughn, B., Santos, A., \& Bost, K. (2008). Secure base representations for both fathers and mothers predict children's secure base behavior in a sample of Portuguese families. Attachment \& Human Development, 10(2), 189-206.

Oppenheim, D. (1997). The attachment doll-play interview for preschoolers. International Journal of Behavioral Development, 20(4), 681-697.

Page, T., \& Bretherton, I. (2001). Mother - and father - child attachment themes in the story completions of pre-schoolers from post-divorce families: Do they predict relationships with peers and teachers? Attachment \& Human Development, 3(1), $1-29$.

Page, T., \& Bretherton, I. (2003a). Gender differences in stories of violence and caring by Preschool Children in post-divorce families: Implications for social competence. Child \& Adolescent Social Work Journal, 20(6), 485-508.

Page, T., \& Bretherton, I. (2003b). Representations of attachment to father in the narratives of Preschool Girls in postdivorce families: Implications for family relationships and social development. Child \& Adolescent Social Work Journal, 20(2), 99-122.

Pierrehumbert, B., Santelices, M., Ibáñez, M., Alberdi, M., Ongari, B., Roskam, I., et al. (2009). Gender and attachment representations in the preschool years: Comparisons between five countries. Journal of Cross-Cultural Psychology, 40(4), 543-566.

Schoppe-Sullivan, S., Diener, M., Mangelsdorf, S., Brown, G., McHale, J., \& Frosch, C. (2006). Attachment and sensitivity in family context: The roles of parent and infant gender. Infant and Child Development, 15(4), 367-385. doi: 10.1002/icd.449

Seabra-Santos, M. J., Simões, M. R., Albuquerque, C. P., Pereira, M. M., Almeida, L. S., Ferreira, C., et al. (2003). Escala de Inteligência de Wechsler para a Idade Pré-Escolar e Primária - Forma Revista (W.P.P.S.I.-R.). In M. M. Gonçalves, M. R. Simões, L. S. Almeida, \& C. Machado (Eds.), Avaliação psicológica: Instrumentos validados para a população (Vol. 1, pp. 197-219). Coimbra, Portugal: Quarteto.

Silva, F., Fernandes, M., Verissimo, M., Shin, N., Vaughn, B. \& Bost, K. (2008). A concordância entre o comportamento de base segura com a mãe nos primeiros anos de vida e os modelos internos dinâmicos no pré-escolar. Análise Psicológica, 26(3), 411-422.
Taylor, S., Klein, L., Lewis, B., Gruenewald, T., Gurung, R., \& Updegraff, J. (2000). Biobehavioral responses to stress in females: Tend-and-befriend, not fight-or-flight. Psychological Review, 107(3), 411-429.

Taylor, S., Lewis, B., Gruenewald, T., Gurung, R., Updegraff, J., \& Klein, L. (2002). Sex differences in biobehavioral responses to threat: Reply to Geary and Flinn (2002). Psychological Review, 109(4), 751-753.

Torres, N., Maia, J., Veríssimo, M., Fernandes, M., \& Silva, F. (2011). Attachment security representations in institutionalized children and children living with their families: Links to problem behavior. Clinical Psychology \& Psychotherapy, 19, 25-36. doi: 10.1002/cpp.739

Trapolini, T., Ungerer, J., \& McMahon, C. (2007). Maternal depression and children's attachment representations during the preschool years. British Journal of Developmental Psychology, 25, 247-261.

Verschueren, K., Marcoen, A., \& Schoefs, V. (1996). The internal working model of the self, attachment, and competence in five-year-olds. Child Development, 67(5), 24932511.

Von Klitzing, K., Kelsay, K., Emde, R. N., Robinson, J., \& Schmitz, S. (2000). Gender-specific characteristics of 5-yearolds' play narratives and associations with behavior ratings. Journal of the American Academy of Child and Adolescent Psychiatry, 39(8), 1017-1023.

Waters, H. S., \& Rodrigues-Doolabh, L. (2004). Manual for decoding secure base narratives. Unpublished manuscript, State University of New York at Stony Brook, Nova York.

Williams, S., \& Blunk, E. (2003). Sex differences in infantmother attachment. Psychological Reports, 92(1), 84-88.

Winnicott, D. W. (1971). Playing and reality. London: Tavistock. Wong, M., Bost, K., Shin, N., Veríssimo, M., Maia, J., Monteiro, L., et al. (2011) Preschool children's mental representations of attachment: Antecedents in their secure base behaviors and maternal attachment scripts. Attachment \& Human Development, 13(5), 489-502. doi: 10.1080/14616734.2011. 602256

Zahn-Waxler, C., Shirtcliff, E. A., \& Marceau, K. (2008). Disorders of childhood and adolescence: Gender and psychopathology. Annual Review of Clinical Psychology, 4, 275-303. 